\title{
Fitting of von Bertalanffy growth model: Stochastic differential equation approach
}

\author{
PRAJNESHU, HIMADRI GHOSH AND N. N. PANDEY* \\ ICAR-Indian Agricultural Statistics Research Institute, New Delhi - 110 012, India \\ *ICAR-Directorate of Coldwater Fisheries Research, Bhimtal, Nainital- 263 136, Uttarakhand, India \\ e-mail: prajneshug@gmail.com
}

\begin{abstract}
In this paper, the well-known von Bertalanffy growth (VBG) model for estimating age-length relationship in fisheries is considered. It is emphasised that nonlinear estimation procedures should be adopted for fitting the von Bertalanffy nonlinear statistical (VBNS) model rather than the age-old Ford-Walford plot. Some limitations of employing VBNS modelling approach are highlighted. Employment of stochastic differential equation (SDE) approach, which does not suffer from these limitations, is advocated for fitting the VBG model. The methodology for fitting the von Bertalanffy SDE (VBSDE) model is described. Relevant computer code for fitting this model is written in SAS package and the same is included as an Appendix. Finally, as an illustration, superiority of VBSDE model over VBNS model for fitting and forecasting purposes is shown for rainbow trout (Onchorhynchus mykiss) age-length data.
\end{abstract}

Keywords: Fish age-length data, SAS software package, Stochastic differential equation, von Bertalanffy growth model

\section{Introduction}

The von Bertalanffy growth (VBG) model plays a very important role in estimating age-length relationship in fisheries, as is evident from the fact that a large number of articles dealing with this model have appeared recently in journals (Menon et al., 2015; Chembian and Mathew, 2016; Ganesh and Chakravarty, 2016; Jayabalan et al., 2016). In the case of fish species from temperate waters, age reading is generally a relatively simple technique, because their otoliths or scales show seasonal rings: one for the summer and one for the winter, which together form an annual ring. Unfortunately, tropical fish species seldom show clear annual rings in their otoliths or scales because strong seasonality is lacking. Consequently, it is difficult to determine their ages. However, daily increments in tropical fish (or even increments caused by a certain food intake) can be detected by a scanning electron microscope. Therefore, continuing to use the age old Ford-Walford plot, which is employed to obtain a quick estimate of maximum length of fish without using age data explicitly, does not make sense (Sparre and Venema, 1998).

Even when fish age data is used explicitly, usual practice to fit VBG model is to apply a transformation to get rid of the inherent nonlinearity of the model and subsequently use the method of least squares for estimation of parameters. As highlighted by Prajneshu (1991), the disturbing feature of this approach is that it is mathematically incorrect and leads to erroneous conclusions. Nevertheless, there may be some justification for employing the above procedure up to, say year 1990, when software for fitting the VBNS model through nonlinear estimation procedures, such as LevenbergMarquardt method were not readily available. But in year 2017, the ground reality is that almost all the standard statistical software packages, such as SAS or PASW contain programs for fitting nonlinear models employing nonlinear estimation procedures. So, now there is absolutely no justification in continuing to adopt the transformation approach. Further, Venugopalan and Prajneshu (1997) advocated to employ VBNS with autocorrelated errors whenever residual analysis indicates that the errors are not independent.

Although the above methodology has served many purposes in the past, it suffers from two main limitations. The first one is that it is applicable only when the data are equidistant. However, collection of growth data over time involves constraints of time, personnel and budget. that do not always satisfy this requirement. For aquacultured fish, getting age-length data at equal intervals may not be difficult but for capture fisheries, this type of data are invariably at unequal intervals. Undoubtedly, in studies with missing data or data at unequal time intervals are potentially informative and precluding such data from analysis could affect conclusions adversely (Dennis and Ponciano, 2014). The other limitation is that, by simply adding an error term, a nonlinear statistical model is not 
capable of describing underlying fluctuations of the system satisfactorily, particularly for longitudinal data. Both the above issues can however, be tackled by employing the more general approach of stochastic differential equations. These are generally obtained by adding a stochastic term to the differential equation form of the deterministic model. Further, in a physical situation, random environmental fluctuations due to variations in the parameters generally occur with great rapidity as compared to the time-scale of growth. Therefore, the stochastic term is generally assumed to be a Gaussian white noise stochastic process. To this end, two types of stochastic calculi due respectively to Stratonovich and Ito (Oksendal, 2003) have been developed in the literature. However, for the present article, both these calculi yield identical results as we are concerned here only with the case of additive noise, which is independent of state variable.

Prajneshu and Venugopalan (1999) developed the methodology for fitting of VBG model in random environment and applied it to pearl oyster (Pinctada fucata) data. However, one limitation of this work was that the methodology was developed and illustrated on the data for the entire group per se as the detailed fish-wise age-length data were not available. Purpose of the present article is to develop the methodology for application of VBSDE model, which is valid when age-length data of individual fish are available.

\section{Materials and methods}

The deterministic von Bertalanffy age-length growth model can be written as:

$$
\mathrm{l}_{\mathrm{t}}=\alpha\left[1-\exp \left\{-\beta\left(\mathrm{t}-\mathrm{t}_{0}\right)\right\}\right]
$$

where $\alpha, \beta, \mathrm{t}_{0}$ represent respectively the ultimate fish length, curvature parameter and initial time-epoch at which fish length is zero. The corresponding VBNS model is obtained by adding an independent and identically distributed (i.i.d.) error term on the right hand side of eq. (1). As advocated by Venugopalan and Prajneshu (1997), the VBNS model should be fitted with autocorrelated errors, if the assumption of i.i.d. errors is violated. Specifically, the VBNS-AR(1) model is given by:

$$
\begin{aligned}
& 1_{\mathrm{t}}=\alpha\left(1-\exp \left\{-\beta\left(\mathrm{t}-\mathrm{t}_{0}\right)\right\}\right)+\varepsilon_{\mathrm{t}} \\
& \varepsilon_{\mathrm{t}}=\rho \varepsilon_{\mathrm{t}-1}+\eta_{\mathrm{t}}
\end{aligned}
$$

It may be pointed out that the VBNS model does not allow prediction of length at every future time-epoch for a continuous-time parameter process. So, differential equation for growth process $l_{t}$ should be considered as stochastic in nature or equivalently the model for changes $\delta l_{t}=l_{t}+\delta t-l_{t}$, rather than $l_{t}$ should be considered. Therefore, following along similar lines as Filipe et al.
(2013), analogous VBSDE model with constant diffusion coefficient is given by:

$$
\mathrm{dl}_{\mathrm{t}}=\mathrm{r}\left(\alpha-\mathrm{l}_{\mathrm{t}}\right) \mathrm{dt}+\sigma \mathrm{dW} \mathrm{t}_{\mathrm{t}}
$$

where $\mathrm{W}_{\mathrm{t}}$ is a Wiener process with variance parameter unity. Eq. (3) is equivalent to:

$$
\operatorname{dexp}(\mathrm{rt}) \mathrm{l}_{\mathrm{t}}=\operatorname{r\alpha exp}(\mathrm{rt}) \mathrm{dt}+\sigma \exp (\mathrm{rt}) \mathrm{dW}_{\mathrm{t}}, \mathrm{l}_{\mathrm{t} 0}=0 .
$$

Integrating both sides and applying Ito calculus, solution of the VBSDE model, given $\mathrm{F}_{\mathrm{tx}}=\left\{\mathrm{l}_{\mathrm{tj}}: \mathrm{j} \leq \mathrm{k}\right\}$ is:

$$
1_{t}=\alpha+\left(1_{t k}-\alpha\right) e^{-r(t-t x)}+\sigma \exp (-r t) \int_{t k}^{t} \exp (r s) d W_{s}
$$

Since age-length data is observed in controlled environment and length data is obtained from age at time-epoch $t=0$, therefore it is of interest to estimate $t_{0}$ in addition to $r, \alpha, \sigma^{2}$. Note that the process $\left(l_{t}: t \geq t_{0}\right)$ is Markovian and stationary with conditional mean $\mu \mathrm{l}: \mathrm{t} \mid \mathrm{t}_{\mathrm{k}}$ and variance $\sigma^{2} \mathrm{t}: \mathrm{t} \mid \mathrm{tk}$ given by:

$$
\begin{aligned}
& \mu 1: t \mid t_{k}=E\left\{1_{t} \mid l_{s}: s \leq t_{k}\right\}=\alpha+\left(1_{t_{k}}-\alpha\right) \mathrm{e}^{-r\left(t-t_{k}\right)} \\
& \sigma_{1: t \mid t_{k}}^{2}=V\left\{1_{t} \mid l_{s}: s \leq t_{k}\right\}=\frac{\sigma^{2}\left(1-e^{-2 r(t-t-k)}\right)}{2 r}
\end{aligned}
$$

Method of maximum likelihood is applied to obtain estimates of parameters. To this end, joint likelihood is expressed in terms of product of conditional likelihoods at time-epoch $\mathrm{t}$ given $\mathrm{F}_{\mathrm{tk}}=\left\{\mathrm{l}_{\mathrm{s}} \mathrm{s} \leq \mathrm{t}_{\mathrm{k}}\right\}$, which are Gaussian with conditional means and variances respectively given by eqs. (6a) and (6b). It may be highlighted that only the VBSDE model is capable of predicting future length at any time-epoch continuously. The optimal (exact) predictor $1_{t}$ of given $\left\{l_{s}: s \leq t_{k}\right\}$ is given by $\mu \mathrm{l}: \mathrm{t} \mid \mathrm{t}_{\mathrm{k}}=\mathrm{E}\left\{\mathrm{l}_{\mathrm{t}} \mid \mathrm{l}_{\mathrm{s}}: \mathrm{s} \leq \mathrm{t}_{\mathrm{k}}\right\}=\alpha+\left(\mathrm{lt}_{\mathrm{k}}-\alpha\right) \mathrm{e}^{-\mathrm{r}(\mathrm{t}-\mathrm{t} \mathrm{k})}$. One may also use naïve approach for prediction of $l_{t}$ by considering the predicted value of $1_{t}$ at some intermediate time-epoch $t^{\prime}$ where $\mathrm{t}_{\mathrm{k}}<\mathrm{t}^{\prime}<\mathrm{t}$. Relevant computer code for fitting the model to data in SAS software package (SAS, 2013). was developed and the same is given as an Appendix. Finally, goodness-of-fit of fish-wise fitted model was assessed by computing the root mean square error (RMSE), given by:

$$
\operatorname{RMSE}=\left[\sum_{\mathrm{t}=1}^{\mathrm{n}}\left(\mathrm{y}_{\mathrm{t}}-\hat{\mathrm{y}}_{\mathrm{t}}\right)^{2} / \mathrm{n}\right]^{1 / 2}
$$

where $\mathrm{y}_{\mathrm{t}}$ and $\hat{y}_{\mathrm{t}}$ denote respectively the observed and fitted values at time $t$ and $n$ indicates the number of time-epochs. Averages of above fish-wise RMSE values would reflect the overall performance of fitted model.

\section{Results and discussion}

As an illustration, age-length data of rainbow trout Onchorhynchus mykiss (Walbaum, 1792), obtained from ICAR-Directorate of Coldwater Fisheries Research (ICAR-DCFR), Bhimtal, India are considered for fitting VBNS and VBSDE models. Specifically, the data comprise lengths $(\mathrm{mm})$ of 30 rainbow trout fish recorded 
under raceway farming conditions on monthly basis for 20 months. Among the freshwater salmonids, rainbow trout is one of the promising cultivable fish species in coldwater and has considerable scope for its expansion in upland region. Being a low volume high value commodity, the trout has good potential for domestic consumption as well as for export. In spite of having excellent positive traits, development and expansion of trout farming has yet to be done on a large scale. This species is native to the Pacific drainages of North America ranging from Alaska to Mexico. However, it is the world's most widely introduced fish species, which is cultured over 100 countries including India. Since early nineties, India has taken up farming of rainbow trout in hills and aquaculture of rainbow trout is gaining significance in the context of income generation, employment opportunities and food security to the people dwelling in hills. The north-western Himalayan region of Jammu and Kashmir and Himachal Pradesh, central Himalaya region of Uttarakhand State, north-eastern region of Sikkim, Arunachal Pradesh and Nilgiries as well as Munnar hills in Peninsular India are potential areas for rainbow trout farming.

The lengths $(\mathrm{mm})$ of each of 30 fish were observed at ages $0,1,2, \ldots, 20$ months. In the first instance, the data up to age 18 months were used for fitting VBNS growth model, while those for ages 19 and 20 months were employed for studying performance of the fitted model for prediction purpose. The parameters were estimated by nonlinear least squares estimation technique using Levenberg-Marquardt optimisation procedure, available in SAS package. Maximum likelihood methodology was employed to estimate parameters and for fitting VBSDE model. After fitting VBNS-AR (1) models to individual fish data, residual analyses were carried out by applying Run test. It is noticed that, except for the fish with serial number 30, all the remaining fitted models do not violate the assumption of independence of error terms at 5\% level of significance. In the second instance, VBSDE models were fitted to individual fish age-length data using the computer code given in the Appendix.

Subsequently, the values were obtained for fitted VBNS and VBSDE models by using respective values for each model and the same are given in Table 1. Evidently, VBSDE model performs better than VBNS model as the fitted values for the former are much closer to actual values than those of the latter. The goodness-of-fit of fitted VBNS and VBSDE models were studied by computing RMSE values using eq. (7) for all the 30 fish, but, to save space, the same for 7 randomly selected fish are presented in Table 2. Again the superiority of VBSDE model over VBNS model is evident. Finally, average of RMSE values for fitted VBSDE models for age-length data of 30 fish is
Table 1. Estimated lengths (mm) at age 0 using fitted VBNS-AR (1) and VBSDE models

\begin{tabular}{llll}
\hline $\begin{array}{l}\text { Fish } \\
\text { no. }\end{array}$ & $\begin{array}{l}\text { Initial length } \\
(\mathrm{mm})\end{array}$ & $\begin{array}{l}\text { VBNS-AR(1) } \\
\text { model }\end{array}$ & $\begin{array}{l}\text { VBSDE } \\
\text { model }\end{array}$ \\
\hline 1 & 6 & $9.25(-0.173)$ & $5.99(-0.190)$ \\
2 & 7 & $6.64(-0.208)$ & $7.31(-0.221)$ \\
3 & 8 & $7.15(-0.218)$ & $7.99(-0.239)$ \\
4 & 7 & $6.19(-0.194)$ & $7.06(-0.214)$ \\
5 & 6 & $5.48(-0.175)$ & $6.42(-0.202)$ \\
6 & 7 & $4.41(-0.167)$ & $6.98(-0.212)$ \\
7 & 6 & $4.50(-0.138)$ & $6.55(-0.199)$ \\
8 & 8 & $7.39(-0.226)$ & $7.94(-0.236)$ \\
9 & 8 & $7.18(-0.217)$ & $7.98(-0.242)$ \\
10 & 7 & $4.58(-0.164)$ & $6.98(-0.212)$ \\
11 & 7 & $6.16(-0.172)$ & $6.76(-0.204)$ \\
12 & 8 & $7.19(-0.147)$ & $7.97(-0.241)$ \\
13 & 6 & $4.70(-0.211)$ & $5.96(-0.188)$ \\
14 & 8 & $7.00(-0.215)$ & $7.99(-0.239)$ \\
15 & 8 & $7.08(-0.122)$ & $7.99(-0.189)$ \\
16 & 6 & $3.96(-0.152)$ & $6.39(-0.200)$ \\
17 & 7 & $4.99(-0.157)$ & $6.96(-0.238)$ \\
18 & 8 & $6.50(-0.131)$ & $7.92(-0.190)$ \\
19 & 6 & $3.60(-0.960)$ & $6.37(-0.215)$ \\
20 & 7 & $5.47(-0.161)$ & $6.99(-0.239)$ \\
21 & 8 & $6.41(-0.202)$ & $7.98(-0.207)$ \\
22 & 7 & $5.90(-0.106)$ & $6.87(-0.183)$ \\
23 & 6 & $5.08(-0.063)$ & $5.79(-0.240)$ \\
24 & 8 & $7.01(-0.153)$ & $7.97(-0.211)$ \\
25 & 7 & $5.24(-0.231)$ & $6.87(-0.241)$ \\
26 & 8 & $3.88(-0.145)$ & $7.97(-0.194)$ \\
27 & 6 & $4.97(-0.267)$ & $5.94(-0.239)$ \\
28 & 8 & $6.68(-0.177)$ & $7.98(-0.213)$ \\
29 & 7 & $6.30(-0.176)$ & $6.99(-0.207)$ \\
30 & 7 & ------- & $6.96(-0.217)$ \\
\hline & 6 & & \\
15 & 6 & &
\end{tabular}

"Figures in brackets indicate $\mathrm{t}_{\mathrm{o}}$ values

Table 2. RMSE values for fitted VBNS-AR (1) and VBSDE models

\begin{tabular}{lll}
\hline Fish no. & VBNS-AR(1) model & VBSDE model \\
\hline 1 & 09.45 & 5.11 \\
2 & 09.00 & 7.27 \\
3 & 09.17 & 7.42 \\
4 & 09.37 & 7.46 \\
5 & 09.19 & 7.82 \\
6 & 09.76 & 8.15 \\
7 & 10.27 & 8.78 \\
\hline
\end{tabular}

computed as $7.01 \mathrm{~mm}$, which being quite low, shows that VBSDE model provides a good fit to the given data. To get a visual idea, the graphs of fitted VBSDE model for 2 randomly selected fish along with data are depicted in Fig. 1. 


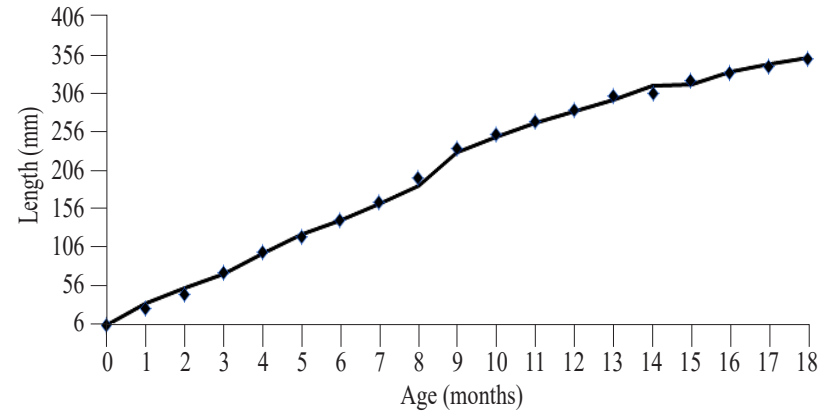

Fig. 1. VBSDE model for Fish 1 along with data

Finally, performances of fitted models were assessed for prediction purpose by computing fish-wise one-step and two-step ahead predictions and then computing root mean square prediction error (RMSPE) values for 30 fish using eq. (7) and the same are reported in Table 3. A perusal of this table also shows that, for given data, performance of VBSDE model even for prediction purposes is much better than that of VBNS model. To get insight, comparison of fitting and forecast values for VBNS and VBSDE models in respect of 2 randomly selected fish are summarised in Table 4, which again reflects superiority of VBSDE model over VBNS model for given data.

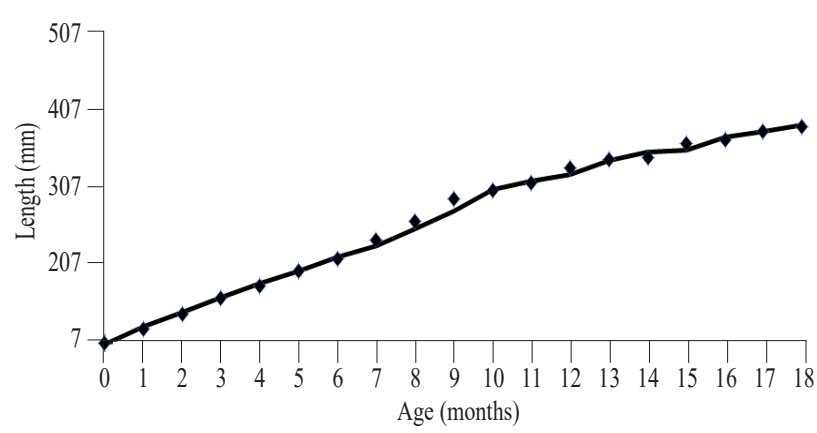

Fig. 2. VBSDE model for Fish 2 along with data

Table 3. RMSPE values for fitted VBSDE and VBNS models for 30 fish

\begin{tabular}{lrllll}
\hline \multicolumn{2}{l}{ VBNS-AR(1) Model } & & \multicolumn{3}{c}{ VBSDE Model } \\
\cline { 1 - 3 } \cline { 5 - 6 } Age 19 & Age 20 & & Age 19 & \multicolumn{2}{c}{ Age 20 } \\
\hline & & & Naive / Exact & Naive & Exact \\
12.83 & 13.81 & & 2.67 & 3.39 & 2.88 \\
\hline
\end{tabular}

In view of the above discussion, it is hoped that in future fishery scientists would start applying VBSDE model to analyse age-length data sets.

Table 4. Comparison of fitting and forecast values for VBNS and VBSDE models in respect of 2 randomly selected fish

\begin{tabular}{|c|c|c|c|c|c|c|c|c|c|c|}
\hline \multirow{3}{*}{ Age } & & & \multicolumn{4}{|c|}{ VBNS-AR(1) model } & \multicolumn{4}{|c|}{ VBSDE model } \\
\hline & \multicolumn{2}{|c|}{ Data } & \multicolumn{2}{|c|}{ Fish 1} & \multicolumn{2}{|c|}{ Fish 2} & \multicolumn{2}{|c|}{ Fish 1} & \multicolumn{2}{|c|}{ Fish 2} \\
\hline & Fish 1 & Fish 2 & Fitting & Forecast & Fitting & Forecast & Fitting & Forecast & Fitting & Forecast \\
\hline 0 & 6 & 7 & 9.25 & - & 4.41 & - & 5.99 & - & 6.98 & - \\
\hline 1 & 29 & 33 & 23.60 & - & 28.69 & - & 36.46 & - & 38.82 & - \\
\hline 2 & 48 & 60 & 54.78 & - & 60.12 & - & 58.28 & - & 63.42 & - \\
\hline 3 & 78 & 86 & 84.37 & - & 89.99 & - & 76.31 & - & 88.97 & - \\
\hline 4 & 106 & 108 & 112.45 & - & 118.36 & - & 104.77 & - & 113.57 & - \\
\hline 5 & 127 & 134 & 139.11 & - & 145.32 & - & 131.33 & - & 134.39 & - \\
\hline 6 & 150 & 154 & 164.41 & - & 170.93 & - & 151.25 & - & 158.99 & - \\
\hline 7 & 176 & 186 & 188.43 & - & 195.26 & - & 173.08 & - & 177.91 & - \\
\hline 8 & 208 & 218 & 211.23 & - & 218.38 & - & 197.74 & - & 208.19 & - \\
\hline 9 & 248 & 258 & 232.86 & - & 240.33 & - & 245.01 & - & 238.47 & - \\
\hline 10 & 268 & 274 & 253.40 & - & 261.20 & - & 266.05 & - & 276.32 & - \\
\hline 11 & 284 & 286 & 272.89 & - & 281.02 & - & 285.02 & - & 291.46 & - \\
\hline 12 & 302 & 312 & 291.39 & - & 299.85 & - & 300.20 & - & 302.82 & - \\
\hline 13 & 321 & 326 & 308.95 & - & 317.73 & - & 317.28 & - & 327.42 & - \\
\hline 14 & 324 & 330 & 325.61 & - & 334.73 & - & 335.31 & - & 340.66 & - \\
\hline 15 & 342 & 354 & 341.43 & - & 350.87 & - & 338.15 & - & 344.45 & - \\
\hline 16 & 354 & 364 & 356.44 & - & 366.21 & - & 355.23 & - & 367.16 & - \\
\hline 17 & 362 & 376 & 370.69 & - & 380.78 & - & 366.61 & - & 376.62 & - \\
\hline 18 & 374 & 384 & 384.22 & - & 394.63 & - & 374.20 & - & 387.98 & - \\
\hline 19 & 382 & 393 & - & 397.05 & - & 407.78 & - & 385.58 & - & 395.54 \\
\hline 20 & 396 & 405 & - & 409.24 & - & 420.27 & - & 393.17 & - & 404.06 \\
\hline
\end{tabular}




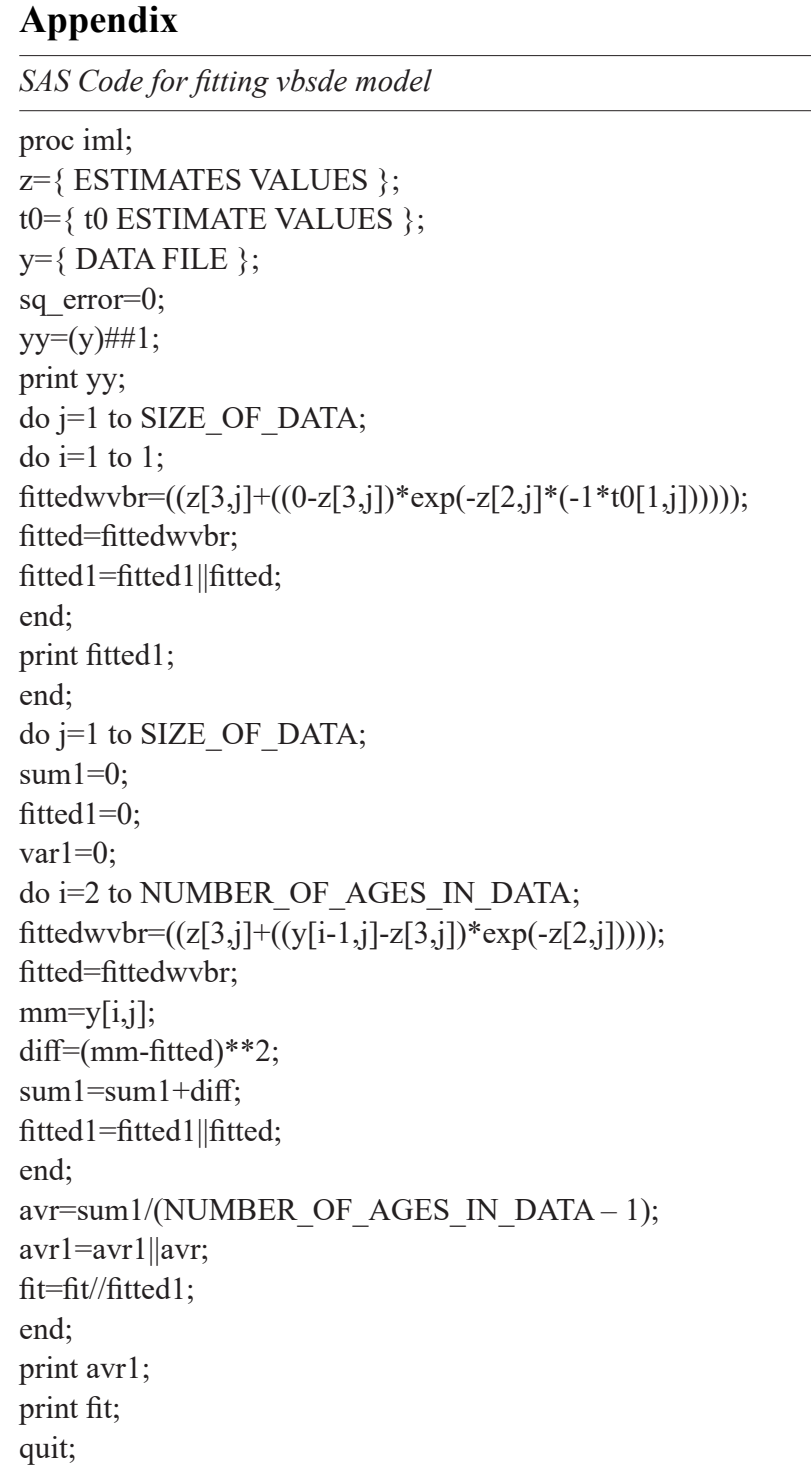

\section{Acknowledgements}

The authors are grateful to Science and Engineering Research Board, New Delhi for providing financial assistance to carry out the above research work under Research Project No. SB/S4/MS/880/2014. Thanks are also due to Ms. Muktha Menon, Scientist, Visakhapatnam Regional Centre of ICAR-Central Marine Fisheries Research Institute, Visakhapatnam, India for stimulating discussions.

\section{References}

Chembian, A. J. and Mathew, S. 2016. Growth and mortality of the oceanic squid Sthenoteuthis oualaniensis (Lesson, 1830) off south-west coast of India. Indian J. Fish., 63(1): 27-34.

Dennis, B. and Ponciano, J. M. 2014. Density dependent state space model for population abundance data with unequal time intervals. Ecology, 95: 2069-2076.

Filipe, P. A., Braumann, C. A., Brites, N. M. and Roquete, C. J. 2013. Prediction for individual growth in a random environment. In: Oliveira, P. E., Temido, M. G., Henriques, C. and Vichi, M. (Eds.), Recent developments in modeling and applications in statistics. Berlin, Springer, p. 193-201.

Ganesh, P. R. C. and Chakravarty, M. S. 2016. Age and growth of the deep water mud shrimp Solenocera melantho (De Man, 1907) off Visakhapatnam coast, India. Indian J. Fish., 63(4): 22-27.

Jayabalan, N., Zaki, S., Al-Marzouqi, A. and Al-Kiyumi, F. 2016. Stock assessment of the Indian mackerel Rastrelliger kanagurta (Cuvier, 1817) from Muscat coast, Oman. Indian J. Fish., 63(4): 28-34.

Menon, M., Maheswarudu, G., Rohit, P., Laxmilatha, P., Das, M. and Rao, K. N. 2015. Biology and stock assessment of the bigeye croaker Pennahia anea (Bloch, 1793) landed along Andhra Pradesh, north-east coast of India. Indian J. Fish. 62(1): 46-51.

Oksendal, B. 2003. Stochastic differential equations: An introduction with applications. Springer, Norway.

Prajneshu 1991. Cautionary note about non-linear models in fisheries. Indian J. Fish., 38(4): 231-33.

Prajneshu and Venugopalan, R. 1999. von Bertalanffy growth model in a random environment. Can. J. Fish. Aquat. Sci., 56: $1026-1030$

SAS 2013. Statistical analysis system, Ver. 9.4. North Carolina.

Sparre, P. and Venema, S. C. 1998. Introduction to tropical fish stock assessment - Part 1: Manual. Food and Agricultural Organisation, Rome.

Venugopalan, R. and Prajneshu 1997. von Bertalanffy growth model with autocorrelated errors. Indian J. Fish., 44(1): 63-67. 